\title{
Neueste kulturlandschaftliche Veränderungen in Ostanatolien, speziell in der Region Kars
}

\author{
Werner Kündig-Steiner
}

Bereits im Jahre 1953 hatte ich Gelegenheit, Ostanatolien, besonders den Raum um den Vansee, zu bereisen. Die Sommerstudienreise 1967 diente vornehmlich zu Vergleichszwecken. Zudem lernte ich zusätzlich die Region zwischen Ararat und der Kolchis vom agrarwirtschaftlichen Standpunkt aus kennen. Auch ein großer Abstecher in Richtung Dreiländerecke Türkei-Persien-Irak, in den Hakkari-Gebirgsraum, erhöhte das Verständnis für jüngste kulturlandschaftliche Veränderungen Ostanatoliens.

Die seit 1929 eingewanderte türkische Bevölkerung jenseits des Euphrat, die heute mit einer kurdischen Minderheit von nahezu zwei Millionen Menschen durchsetzt ist, war von der westlich-industriellen Wirtschaft und Kultur etwa drei Jahrzehnte lang abgesperrt. Das engere Ostanatolien - rund dreimal so groß wie die Schweiz - blieb besonders in technischer Beziehung gänzlich unterentwickelt; es ist auch heute ausgesprochener Gebirgsraum und agrarwirtschaftlich seit Jahrhunderten auf Selbstversorgung eingestellt. Alle neuzeitlichen Konsumgüter werden aus der westlichen Türkei oder gar aus dem Ausland herangeschafft. In den Regierungskreisen von Ankara gesteht man freimütig, daß Ostanatolien in allen nationalen Wirtschaftsplänen, auch im laufenden Fünfjahresplan, viel zu wenig berücksichtigt wurde. Gerade jetzt zögert man sehr, größere Summen von den Entwicklungshilfegeldern ausschließlich für diesen Landesteil abzuzweigen; es gäbe noch viel vordringlichere Aufgaben, sagt man in Ankara.

Das strategisch äußerst wichtige östliche Anatolien - ein Gebirgsland mit engen Durchbrüchen durch gewaltige Basaltdecken - ist in der Nachkriegszeit durch die Gegenwart eines großen, stehenden Heeres gekennzeichnet, das allein in dieser Region etwa eine halbe Million Mann zählen mag. Die völlige Durchsetzung mit Garnisonen aller Art erlaubt dem Geographen von typischen "Militärlandschaften" zu sprechen, die sich gleichzeitig sehr scharf von den reinen Agrarlandschaften abheben. Die stärksten Nutznießer dieses Nebeneinanders oder dieser «Scheinsymbiose» sind mehrere rapid wachsende Hochland-Provinzstädte, besonders Erzurum und Van. Diese Städte offenbaren koloniale Wachstumserscheinungen. In den sich schnell wandelnden Hauptstraßen verschwinden alle bäuerlich anmutenden Bauten, und bereits machen zweistöckige
Bauten einzelnen Hochhäusern Platz. Die «Skyline» ist daher außergewöhnlich bewegt: im Zentrum erscheinen die ersten zementierten Wohnblöcke, sodann ungewöhnlich großzügig ausgedachte Hotelbauten, plätzebeherrschende Autoservicestationen und anstelle der Kramladen auf bestimmte Branchen spezialisierte Fachgeschäfte. Bei einem Rundblick über die Stadt ist man von der Verdichtung des Baumbestandes im Innern des Straßengefüges überrascht, viel mehr noch über die radiale Ausweitung des immer an Fließwasser gebundenen Pappel-Grüngürtels: Der Oasencharakter erscheint viel ausgeprägter als je zuvor, das Steppenbild verschwindet.

Auch auf dem Dorf weitab der Stadt sind kulturlandschaftliche Veränderungen leicht feststellbar. Da tauchen erstmals Wohngebäude mit Ziegelsteinwänden auf; seit einigen Jahren überraschen ganz moderne Schulhausbauten. Sehr charakteristisch sind auch die neuen, vom staatlichen Gesundheitsdienst errichteten dörfischen Krankenstationen, verbunden mit Wohnungen für das Personal. Zahlreicher als je erscheinen Moschee-Neubauten.

In Ostanatolien hat sich das Straßennetz im Laufe des letzten Jahrzehntes ganz allgemein wesentlich verbessert. In intensivem Ausbau befindet sich zur Zeit die sogenannte Cento-Straße, die von Van aus über Oezalp die Verbindung mit Täbris in Persien herstellt. Parallel führt die neue Cento-Eisenbahnlinie, die schon im Sommer 1969 eröffnet wird. Mit Hilfe einer modernen Eisenbahnfähre wird man das Binnenmeer von Van überqueren und in der Folge Schwertransporte bis an die persische Grenze ausführen können. Allmählich beginnt sich längs dieser schmalen Furche eine mittelanatolische Bevölkerungsschwerlinie von Kayseri über Malatya-Elazig-Mus-Tatvan und über Van hinaus abzuzeichnen.

In denselben verkehrsgeographischen Problemkreis gehört der vor gut einem Jahre beendete Umbau der russischen Breitspur auf Normalspur längs der Strecke Sarikami-Kars mit Fortsetzung zur sowjetrussischen Grenze bei Leninakan. Damit ist die Region Kars eisenbahntechnisch endlich an die Hauptstadt Ankara angeschlossen, fast ein halbes Jahrhundert nach der Schaffung des modernen Türkeistaates. Als Folge setzte bereits 1967 ein geringer Touristenverkehr zwischen Erzurum und Tiflis ein, der vorwiegend aus Amerika-Armeniern be- 


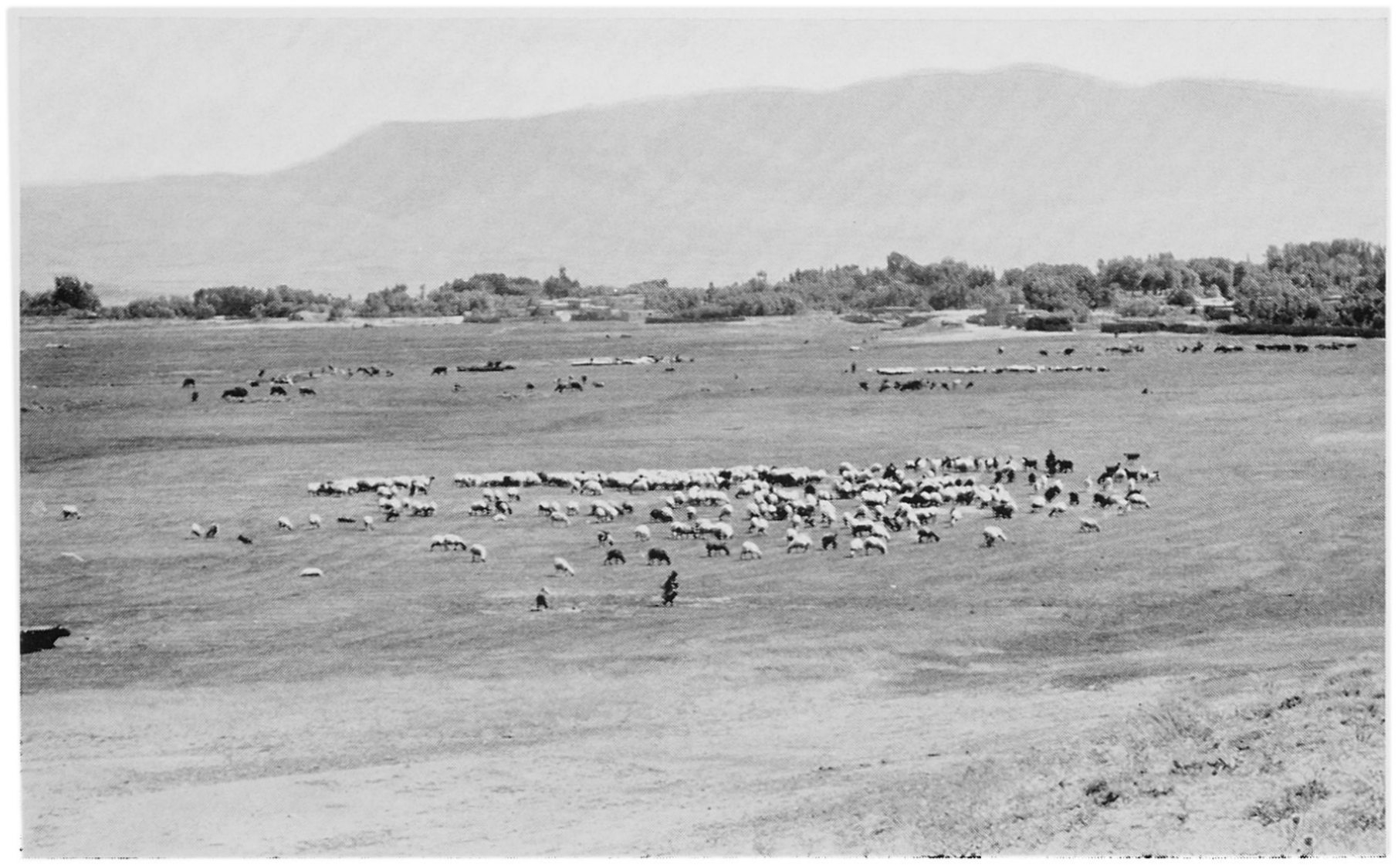

Ostanatolischer Landschaftstyp. An der künftigen CENTO-Bahnlinie, östlich von Van; bei Erçek. Talauen-Kultursteppe. Dorfsiedlung im Grünstreifen. Am Gebirgshang buschiger Sekundärwald. Aufnahme August 1967, Verfasser

stand. Beigefügt sei, daß die nun zwischen den zwei Längseisenbahnlinien liegende, alte Transitstraße von Trapezunt (Trabzon) über Erzurum, am Ararat vorbei, Richtung Teheran dennoch in steigendem Maße als einflußreiche Kulturträgerin ostwärts bis über die Landesgrenze hinaus, weit nach Persien hinein, zu wirken vermag.

Ein Novum in der Erschließung der Karsregion ist jene wildromantische Verbindungs-Gebirgsstraße zwischen Ararat-Hochland und der Kolchis, das heißt zwischen Kars und dem kleinen Hafen Hopa, südwestlich Batum, am Schwarzen Meer. Sie ist stiickweise, wie etwa im Tal des tiefeinschneidenden Çoruh-Flusses, beinahe autobahnartig ausgebaut, aber anderseits doch schwer behindert durch den Yalnizçan-Paß, der $2650 \mathrm{~m}$ ü. M. erreicht. Dieser im Ausbau befindliche Gebirgspaß ist ein Ausschnitt der strategisch bedeutsamen Grenzlandstraße vom Schwarzen Meer über Kars und Van bis an den Tigris. Er dient heute mehr als je zuvor dem Güteraustausch zwischen den östlichsten, schwarzmeerischen Häfen und der viehwirtschaftlich äuBerst produktiven Region um Kars. Da diese Verkehrsader die noch verbliebenen prächtigen, pontischen Nadelhochwälder durchquert, besteht durchaus die Gefahr, daß in der Folge der Wald stärker als bisher gelichtet, aber trotz Forstgesetz nicht genügend ergänzt und gepflegt wird.
Der Geograph kann nicht übersehen, daß sich auch in der Osttürkei seit Jahren der Ackerbau auf Kosten der Weidwirtschaft sehr stark ausgedehnt hat. Diese flächenhafte Ausdehnung gilt weniger für die schon immer stark unter dem Pflug gestandenen mineralreichen, altvulkanischen Böden, sondern ganz allgemein für die guten Weidegründe in den Randlandschaften der Hochlandbecken. Immerhin ist die Motorisierung im Ackerbau in Ostanatolien noch keineswegs so weit vorangetrieben wie etwa in der zentralen Türkei oder gar im Tiefland des Euphrat und Tigris. Leider ist man in der Türkei - auch in Persien - im Umpflügen von trockenen Grassteppen weit über das hinausgegangen, was im Interesse der Erhaltung nicht allein der Fruchtbarkeit, sondern auch im Hinblick auf die bloße Masse des Bodens wünschenswert ist. Denn auf dem zu Unrecht gepflügten Land macht sich heute eine erschreckende Bodenerosion bemerkbar. Wenn es nicht gelingt, dieses nicht natürliche Ackerland in Weideland zurückzuverwandeln, so wird in absehbarer Zeit vielfach nur der nackte Fels übrigbleiben.

Auf Grund einer Untersuchung der FAO sollten daher in der Türkei etwa vier Millionen Hektaren "widerrechtlich» gepflügte Grassteppe in Weideland zurückverwandelt werden, größtenteil mit Hilfe von 
Terrassierung und Anpflanzung von Baumreihen. Je nach persönlicher Initiative des Vali (Bezirksgouverneur) wurden sporadische Sanierungsversuche in Angriff genommen.

An dieser Stelle sei ein klimatologischer Hinweis eingeflochten. In der Karser Region ist der Winter - einfach ausgedrückt - äußerst hart; Minimaltemperaturen von $-35^{\circ}$ bei trockener Luft sind keineswergs außergewöhnlich. Anderseits ist es hier im Vorsommer wesentlich feuchter als etwa um Erzurum oder gar Van. In den zwischen 2000 und $2200 \mathrm{~m}$ ü. M. gelegenen Karser Hochlandbecken, die rundum von Dreitausendern überragt werden, schlägt sich viel Feuchtigkeit aus der nahen Kolchis und auch von der weiter entfernten Kaspisee her ab. Noch im Juni und Juli können nächtliche Minimaltemperaturen bis nahe an den Gefrierpunkt sinken. Wegen der starken Einstrahlung eignen sich die im allgemeinen ziemlich nebelreichen Karser Hochlandbecken schon von Natur aus nicht nur für Naturwiesen, sondern noch mehr für sehr ergiebige Futtergrasäcker. Es zeigen sich hier auffällige Parallelen zu den bevorzugten Hochweiden unserer Bündner Alpen. Wie sehr die Bevölkerung der Karser Region von der Milch lebt, zeigt der Jahresverbrauch je Einwohner: Er steht auf rund $400 \mathrm{~kg}$, im Gegensatz zu Istanbul, wo man nur $18 \mathrm{~kg}$ konsumiert. In der Karser Region war und ist die Milchwirtschaft - relativ betrachtet - erstrangig. Hier liefern gute Milchkühe im Hochsommer bis 121 täglich, das heißt vier- bis fünfmal mehr als im trockenen Zentralanatolien. Sehr nachteilig bleibt freilich, daß sich die Tiere über den Winter «halbwegs durchhungern" müssen.

Ein Großteil der Milch wird bis heute in privaten Käsereien verarbeitet, vorwiegend $\mathrm{zu}$ den etwa $100 \mathrm{~kg}$ schweren, wagenradgroßen "Gravyar», einer Art Emmentaler. Aus einer Mischung von Kuhund Schafmilch entsteht der 10-kg-Kaschar-Käse. der früher gelegentlich in die Sowjetunion exportiert wurde. In der Karser Milchwirtschaft drängt sich eine neuzeitliche, industrielle Verwertung der regionalen Milchschwemme seit Jahren auf. Weil es in den türkischen Städten an genügend guter einheimischer Frischmilch, an Milchpulver, Butter und Markenkäse fehlt, erfährt sie eine neue Planung. So sind seit 1963 die Schweiz — über ihr eigenes Entwicklungshilfeprogramm - sowie die UNICEF am Aufbau einer hochmodernen und pionierhaften Milchpulverfabrik in Kars beteiligt. Dieser Großbetrieb - kombiniert mit einer Molkereischule unter Schweizer Leitung - wird einmal bis 1200001 täglich zu verarbeiten vermögen. Die Großanlage ist im Rohbau erstellt und soll im Sommer 1968 in einer ersten Stufe betriebsbereit sein.

Von größter Veränderungskraft im Bild der ostanatolischen Kulturlandschaft ist die wissenschaitlich betreute Bewässerungswirtschaft. Immer deut- licher beginnen sich heute jene Gebiete abzuzeichnen, die sich schon von Natur aus zur wasserbaulichen Landwirtschaft am besten eignen. Es ist daran erinnert, daß die ostanatolischen Hochebenen nicht allein auf $1700-1800 \mathrm{~m}$ ü. M., sondern vielfach auch in 2000 und $2200 \mathrm{~m}$ ü. M. liegen. Eine Sondersituation bietet die Talebene des Aras, am Nordfuß des Ararat. In dem sehr niederschlagsarmen Raum, in welchem der Jahresniederschlag bis auf $300 \mathrm{~mm}$ absinkt, entdeckt man heute sehr beachtenswerte, moderne Bewässerungsanlagen, sowohl auf türkischer wie auch auf sowjetrussischer Seite. Hier ist es möglich, trotz einer latenten Gefahr zur Bildung von Kälteseen in typischen Hochland-Binnenräumen, subtropische Pflanzen, wie z. B. Reis oder Levante-Baumwolle sowie spezielle Obstbaumkulturen, auf ausgedehnten Flächen mit leichten Böden einzuführen.

Auch in der Umgebung des Vansees - er ist leider sodahaltig und daher fast fischlos - erfährt man besonders deutlich, wie die Bewässerungswirtschaft die Grundlage für eine harmonisierte, agrare Kulturlandschaft werden könnte. $20 \mathrm{~km}$ südlich der Stadt Van ist gegenwärtig ein Bewässerungskanal im Ausbau, der teilweise noch auf urartäischen Anlagen des 9. und 8. Jahrhunderts vor Christus beruht und wohl auch von Byzantinern und Armeniern benützt wurde.

Bekanntlich erreichten die Urartäer einen sehr hohen Stand der Bewässerungstechnik; man braucht nur ihren Wohnsitzen nachzugraben, um auch in der Gegenwart zu erfahren, wo günstige Bewässerungsfelder liegen müßten. Auch im gebirgigen Ubergangssaum zwischen der feuchten Kolchis und dem trockenen, kalten Kuruçay-Tal, das nach Transkaukasien ausmündet, ist die Bewässerungswirtschaft auf weite Strecken traditionell. Ihre Kanalanlagen lassen sich sehr wohl mit den «bisses» im Wallis vergleichen.

Ostanatolien kann immer noch als gänzlich industrielos bezeichnet werden, sofern man von einigen wenigen Konsumgüterbetrieben im Hochlandzentrum Erzurum, vom Kupfererz-Aufbereitungswerk Morgul, von größeren Getreidemühlen und Tabakbetrieben, auch von der im Bau befindlichen Zementfabrik bei Van, sowie vom Sonderfall der Teefabriken an der östlichen Schwarzmeerküste absieht. - Eine plötzlich durchbrechende, allgemeine Industrialisierung wird vorläufig nicht stattfinden.

Es ergibt sich die Folgerung, daß Gesamt-Ostanatolien eine besondere Forschungsstelle nötig hat, die speziell dem wirtschaftlichen Aufbau dieses Raumes dienen sollte. An einer wirtschaftlich starken und vor allem zivilisatorisch solid untermauerten Türkei - zu der auch Ostanatolien gehört sind wir Europäer nicht nur bloß interessiert, sondern wir sind mit ihr je länger, desto mehr auch schicksalhaft verbunden. 